\title{
A Load-Share Reliability Model under the Changeable Piecewise Smooth Load
}

\author{
Sergey V. Gurov and Lev V. Utkin \\ Department of Control, Automation and System Analysis, St.Petersburg State Forest Technical University, Institutsky per. 5, \\ 194021 St.Petersburg, Russia
}

Correspondence should be addressed to Lev V. Utkin; lev.utkin@gmail.com

Received 11 May 2014; Revised 27 August 2014; Accepted 1 September 2014; Published 11 September 2014

Academic Editor: Luigi Portinale

Copyright (C) 2014 S. V. Gurov and L. V. Utkin. This is an open access article distributed under the Creative Commons Attribution License, which permits unrestricted use, distribution, and reproduction in any medium, provided the original work is properly cited.

\begin{abstract}
A new load-share reliability model of systems under the changeable load is proposed in the paper. It is assumed that the load is a piecewise smooth function which can be regarded as an extension of the piecewise constant and continuous functions. The condition of the residual lifetime conservation, which means continuity of a cumulative distribution function of time to failure, is accepted in the proposed model. A general algorithm for computing reliability measures is provided. Simple expressions for determining the survivor functions under assumption of the Weibull probability distribution of time to failure are given. Various numerical examples illustrate the proposed model by different forms of the system load and different probability distributions of time to failure.
\end{abstract}

\section{Introduction}

A lot of available methods and models of the system reliability do not take into account a very important factor which impacts on the real system reliability behavior and takes place in most applications. This factor is a change of working conditions of systems that is caused by a change of the load on the system and its components. A clear example illustrating the impact of the changeable load is provided by Kvam and Peña [1]. The authors of this work describe a large structure supported by welded joints. The structure fails only after a series of supporting joints fail. The failure of one or two welded joints in a bridge support, for instance, might cause the stress on remaining joints to increase, thus causing earlier subsequent failures.

This is an example of a load share model, where component failure rates depend on the working or failed states of other components in the system. According to the load share model for a multi-unit system, failure of a unit may put additional load on the surviving units and may reduce the residual time to system failure. On the other hand, the failure of a unit may release extra resources to other units and may increase the time to system failure. The first case leads to the increased failure rate. The second case corresponds to the reduced failure rate.

Another interesting application example of the changeable load is electrical networks whose load strongly depends on outdoor temperature and directly leads to the reliability behavior changes. For example, in Lapland (Finland), where deviations in outdoor temperature are large, the load increase may be up to $100 \%$ compared to the load at normal temperature [2]. Valor et al. [3] indicate that the electricity load shows maximum values in winter and summer and minimum values in spring and autumn. As pointed out by Tanrioven and Alam [4], studies that quantify power system reliability are often limited to constant transmission rates, covering two weather conditions, namely, normal and adverse weather. Therefore, it is necessary to develop methods of reliability analysis which could take into account the load changes.

One of the pioneering works devoted to load-share models applied in the textile industry was proposed by Daniels [5] in 1945. Daniels originally adopted the load-share model to describe how the strain on yarn fibers increases as individual fibers within a bundle break. Last decades, many authors contribute to the load-share models, for instance, Bebbington et al. [6], Deshpande et al. [7], Lee et al. [8], Kim 
and Kvam [9], Lynch [10], Ross [11], Shao and Lamberson [12], Stefanescu and Turnbull [13], and Yang and Younis [14].

A lot of papers are devoted to statistical analysis of the load-share parameters [15-18]. This is only a small part of many papers devoted to load-share models that talks about their importance and applicability.

One of the most important questions of the load-share model is the rule that governs how failure rates change after some components in the system fail or after changing the internal and external loads. This rule depends on the reliability application and on the system reliability behavior. In fact, a system reliability model is directly defined by the model of the load changes or the load function. We select three kinds of the load functions: piecewise constant, continuous, and piecewise smooth.

The discrete load-share reliability models with the piecewise constant load have been studied by Gurov and Utkin [19]. These models are discrete because the load changes occur step-wise at discrete time instances. In contrast to many available models, the proposed models exploit an important assumption which defines these models. This assumption is the so-called "condition of the residual lifetime conservation" of a system, which is equivalent to the condition of continuity of the cumulative distribution function of time to failure or the reliability (survivor) function.

Suppose that the load is changed at deterministic time moments $t_{1}, t_{2}, \ldots, t_{n-1}$. The load rate $k(t)$ is a function of time whose properties impact on the system reliability measures. In fact, the function $k(t)$ totally defines the loadshare reliability behavior of a system. A case when the function $k(t)$ is a piecewise constant function has been studied in [19].

Another kind of load-share reliability models deals with systems functioning under continuously changed conditions, for instance, under changes of the temperature around the systems (see examples of electrical networks). In this case, the load factor changes continuously, that is, the load rate $k(t)$ is a continuous function. The systems with this load behavior have been studied by Gurov and Utkin [20]. The main assumption accepted in reliability analysis of the systems is again the condition of the residual lifetime conservation.

However, we can always meet applications where the load cannot be described in one of the above forms. It has a more complex behavior which has to be taken into account by means of a corresponding reliability model. Therefore, we extend the above special cases on a case of arbitrary piecewise smooth load function. In other words, we analyze systems whose reliability behavior can be modeled by the piecewise smooth function $k(t)$. The proposed models with the piecewise smooth load can cover a lot of real applications.

Let $P(t)$ be the reliability of a system under normal (or initial) conditions of its usage. The main problem of the system analysis is to determine the reliability of the system by taking into account the possible changes of the load. Our initial information about the changeable load will be represented by means of the load rate as a measure of the load. In order to take into account the changeable conditions, we define another measure $P_{c}(t)$. A method for computing the function $P_{c}(t)$ under the piecewise smooth load is proposed in the paper. Moreover, we investigate in the paper how types of probability distributions of time to failure impact on the reliability measures of the system with different types of the piecewise smooth load rate functions.

The paper is organized as follows. In Section 2, we consider a discrete load-share model and introduce the condition of the residual lifetime conservation of the system. Section 3 provides the reliability analysis of systems with continuous changes of load. The main results concerning the system reliability with the piecewise smooth load and a general algorithm for computing reliability measure are given in Section 4. A special case when the time to failure of a system is governed by the Weibull probability distribution is studied in Section 5. Some expressions for reliability analysis are provided in the explicit form in this section. Numerical examples illustrating the applicability of the proposed model to systems with the monotone load by different probability distributions of time to failure are given in Section 6. Similar numerical examples considering the nonmonotone load are given in Section 7. Comparison of systems under piecewise constant and smooth loads is carried out in Section 8. Conclusion remarks can be found in Section 9.

\section{Discrete Load-Share Models}

We have introduced in the previous section two important survivor functions $P(t)$ and $P_{c}(t)$ which correspond to the reliability behavior under normal or initial conditions and under changeable conditions, respectively. It is assumed that the function $P(t)$ is known or can be computed by means of known methods. The main question of the reliability analysis considered in the paper is how to compute the survivor function $P_{c}(t)$. An answer to the question depends on many factors including the behavior of the load, the behavior of failure rates under changeable condition, and so forth. In order to illustrate these factors and to show a simplest link between the survivor functions $P(t)$ and $P_{c}(t)$, we consider an illustrative example of a parallel system consisting of $n$ units such that $n-1$ units are redundant, which can be analyzed as a discrete load-share model. After failure of a unit, other units are under the increased load which can be measured by the load factor or the load rate denoted as $k$. Note that $k=n /(n-1)$ for the considered parallel system. After failure of the second unit, the load factor becomes $k=n /(n-2)$. After failure of the $i$ th unit, the load factor becomes $k=n /(n-i)$. Finally, after failure of $n-1$ units, the load factor is $k=n$.

Let us consider first a case when the load on a system changes only once at time $t_{1}$ such that the load rate changes from the initial (normal) value 1 before time $t_{1}$ to some new value $k$ corresponding to the load after time $t_{1}$. Let $P_{k}(t)$ be the reliability of the system under condition that the load has changed and became $k$. In fact, the survivor function $P_{k}(t)$ is defined under condition that the system had the load $k$ since time $t=0$. It should be noted that the function $P_{k}(t)$ is auxiliary. It is used in order to get the survivor function $P_{c}(t)$. The main idea underlying the computation of the reliability measure $P_{c}(t)$ after the load changes is the so-called condition 
of the residual lifetime conservation of the system; namely, it can be written as follows:

$$
P_{c}(t)= \begin{cases}P(t), & \text { if } t<t_{1}, \\ P_{k}(t-x), & \text { if } t \geq t_{1},\end{cases}
$$

where the value of the shift $x$ is chosen in such a way that $P_{c}(t)$ is continuous (without jumps).

The condition of the residual lifetime conservation is equivalent to the condition of continuity of the cumulative distribution function of time to failure or the reliability (survivor) function. This condition is natural in most systems. Jumps of a distribution function take place in its approximations. They can be regarded as a mathematical abstraction that can hardly anyhow satisfactorily model any distribution used in reliability theory. To our knowledge, there is no explicit formulation of the condition of the residual lifetime conservation in the reliability literature. However, the reliability analysis of some typical load-sharing systems coincides with the results obtained by Gurov and Utkin $[19,20]$.

The value of the shift $x$ under the condition of the residual lifetime conservation can be also interpreted as follows. The area under the probability density function $f(t)=-P^{\prime}(t)$ in interval $\left[t_{1}, \infty\right)$ is equal to the area under the probability density function $f_{k}(t)=-P_{k}^{\prime}(t)$ in interval $\left[t_{1}-x, \infty\right)$.

Rather simple expressions for $P_{k}(t)$ can be obtained if we link the load rate $k$ and the system failure rate. We have mentioned that one of the crucial questions of load-sharing models defining the reliability analysis is a rule that governs how failure rates are changed after changing the loads. This rule depends on the reliability application and on the system reliability behavior. We suppose that the load increases by $k$ times as the system failure rate increases by $k$ times. Then there holds

$$
P_{k}(t)=P^{k}(t)
$$

It should be noted that this rule totally corresponds to the parallel system reliability behavior considered above.

A case when the load changes occur step-wise at discrete time instances $t_{1}, t_{2}, \ldots, t_{n}$ and the loads between these instances are constant has been studied in [19]. The load rates at these instances are $k_{1}, k_{2}, \ldots, k_{n}$, respectively. If we assume $t_{0}=0, t_{n+1}=+\infty, k_{0}=1$, then the reliability of a system, taking into account the changeable load, is

$$
P_{c}(t)=P_{k_{i}}\left(t-x_{i}\right), \quad t_{i} \leq t<t_{i+1}, i=0,1,2, \ldots, n .
$$

The function $P_{k_{i}}(t)$ is the reliability function of the system under condition that the corresponding load rate is $k_{i}$. Parameters $x_{i}, i=1,2, \ldots, n$, are computed by means of the recurrent algorithm from the following equation:

$$
P_{k_{i}}\left(t_{i}-x_{i}\right)=P_{k_{i-1}}\left(t_{i}-x_{i-1}\right), \quad x_{0}=0 .
$$

So, if we find the parameters $x_{i}$ for all $i=1,2, \ldots, n$, we can compute the function $P_{c}(t)$.

\section{Continuous Load-Share Models}

Now we briefly consider another case when the load is continuous, that is, the function $k(t)$ is continuous. This case has been studied in detail by Gurov and Utkin [20].

Let $k(t)$ be a differentiable function of time characterizing the load rate such that $k(0)=1$. This is an analogue of the normalizing condition which means that the system starts working under a normal load condition. In order to analyze the system reliability by continuous function $k(t)$, we will use the well-known failure rate function $\lambda(t)$ and the cumulative failure rate function $\Lambda(t)$ which is expressed through the function $\lambda(t)$ as follows:

$$
\Lambda(t)=\int_{0}^{t} \lambda(\tau) d \tau
$$

Then the system reliability function can be defined as $P(t)=e^{-\Lambda(t)}$.

We again assume below that the change of the load by $k$ times leads to change of the system failure rate by $k$ times. Then there holds $P_{k}(t)=P^{k}(t)$. It follows from [20] that the reliability function of the system, taking into account the continuously varying load, is

$$
P_{c}(t)=P_{k(t)}(t-x(t)) .
$$

The initial condition here is $x(0)=0$. The function $k(t) \equiv$ 1 corresponds to the lack of load. The last expression for $P_{c}(t)$ can be regarded as a continuous analogue of the expression $P_{c}(t)=P_{k_{i}}\left(t-x_{i}\right)$ given for the discrete models. One can see that a finite set of shifts $x_{i}, i=1,2, \ldots, n$, is replaced by the function $x(t)$.

It should be noted that a survivor function of the system time to failure is totally defined by its cumulative failure rate function. This implies that the function $P_{c}(t)$ can be computed if we find how the cumulative failure rate under the changed load denoted by $M(t)$ depends on the function $\Lambda(t)$. It is proved in [20] by using the condition of the residual lifetime conservation that the cumulative failure rate $M(t)$ is expressed through the function $\Lambda(t)$ as follows:

$$
M(t)=k(t) \Lambda(t-x(t)) .
$$

According to [20], the shift function $x(t)$ can be found as a solution of the differential equation

$$
x^{\prime}(t)=\frac{k^{\prime}(t)}{k(t)} \frac{\Lambda(t-x(t))}{\lambda(t-x(t))} .
$$

If the system is under the continuously varying load with the load rate $k(t)$, then the system reliability function is

$$
P_{c}(t)=e^{-M(t)}=e^{-k(t) \Lambda(t-x(t))} .
$$

It follows from the above that the shift function $x(t)$ depends on the system lifetime probability distribution under the normal working state (the cumulative failure rate $\Lambda(t)$ and the failure rate $\lambda(t))$. It also depends on the system load and the load rate function. 
The above differential equation can be solved in the explicit form only for some special cases. Generally, a corresponding numerical method is required for computing its solution.

Let us consider an important special case when the time to failure under normal conditions of functioning is governed by the exponential distribution with the failure rate $\lambda(t)=$ $\lambda$. Then $\Lambda(t)=\lambda t$. Let us introduce the cumulative load function $K(t)=\int_{0}^{t} k(\tau) d \tau$. Then the above differential equation has the solution

$$
x(t)=\frac{(t k(t)-K(t))}{k(t)} .
$$

This implies that the system under the continuous load $k(t)$ has the reliability function

$$
P_{c}(t)=e^{-\lambda K(t)} .
$$

Other special cases can be found in [20].

\section{Reliability Analysis of a System under the Piecewise Smooth Load}

Now we study a general case when the load can be described by the piecewise smooth function $k(t)$. This case extends the above considered discrete and continuous load-share models. The main difficulty of studying the general case is that the function of load $k(t)$ may have jumps or points of discontinuity.

Let the function $k(t)$ be continuously differentiable on the positive half except for a finite or countable set of points $t_{1}, t_{2}, \ldots, t_{m}$ at which it has discontinuities of the first kind. There are finite bounds at these points to the left $k\left(t_{i}-0\right)$ and to the right $k\left(t_{i}+0\right)$.

Then the cumulative failure rate $M(t)$ corresponding to the load rate $k(t)$ is of the form

$$
M(t)=k(t) \Lambda(t-x(t)),
$$

where $\Lambda(t)$ is the cumulative failure rate function under a "normal" load condition; $x(t)$ is a piecewise smooth function having the following properties:

(i) it satisfies the differential equation in interval $\left(t_{i-1}, t_{i}\right)$

$$
x^{\prime}(t)=\frac{k^{\prime}(t)}{k(t)} \frac{\Lambda(t-x(t))}{\lambda(t-x(t))}
$$

under initial conditions $x\left(t_{i-1}\right)=x\left(t_{i-1}+0\right), i=$ $1,2, \ldots, m$.

(ii) the limit of the function $x(t)$ to the right at the discontinuity point $t_{i}$ is determined by using the bound to the left from the equation

$$
\begin{array}{r}
k\left(t_{i}+0\right) \Lambda\left(t_{i}-x\left(t_{i}+0\right)\right)=k\left(t_{i}-0\right) \Lambda\left(t_{i}-x\left(t_{i}-0\right)\right), \\
i=1,2, \ldots, m .
\end{array}
$$

For generality, we use notation $t_{0}=0, x_{0}=0$.

On the one hand, one can see that expressions (12) and (13) coincide with the corresponding expression for $M(t)$ and the corresponding equation for the shift function $x(t)$, respectively, obtained for the continuous load-share models. However, there are discontinuity points $t_{i}$ which significantly complicate solution of the differential equation (13).

It should be noted that this case is not a combination of the discrete and continuous load-share models considered in the previous sections. It can be regarded as an extension of the continuous load-share model. At the same time, one can see that the discrete model is a special case of models with the piecewise smooth load when $k(t)$ is constant between discontinuity points $t_{i}$. The continuous model can also be regarded as a special case of the considered generalization when $m=0$; that is, there are no discontinuity points of the function $k(t)$.

Below we provide a general algorithm for solving (13).

Step 1 . We solve Cauchy problem (13) in interval $\left(0, t_{1}\right)$ under initial condition $x(0)=0$ in order to find the function $x(t)$ in this interval. Then we compute the limit of the function $x(t)$ to the left $x\left(t_{1}-0\right)$. The limit $x\left(t_{1}+0\right)$ of the function $x(t)$ to the right at point $t_{1}$ is computed by solving (14) by $i=1$.

Step 2. We solve Cauchy problem (13) in interval $\left(t_{1}, t_{2}\right)$ under initial condition $x\left(t_{1}\right)=x\left(t_{1}+0\right)$ in order to find the function $x(t)$ in this interval. Then we compute the limit of the function $x(t)$ at time $t_{2}$ to the left $x\left(t_{2}-0\right)$. The limit $x\left(t_{2}+\right.$ 0 ) of the function $x(t)$ to the right at point $t_{2}$ is computed by solving (14) by $i=2$.

Step 3- $(m-1)$. are repeated in the same way.

Step $(m)$. We solve Cauchy problem (13) in interval $\left(t_{m-1}, t_{m}\right)$ under initial condition $x\left(t_{m-1}\right)=x\left(t_{m-1}+0\right)$ in order to find the function $x(t)$ in this interval. Then we compute the limit of the function $x(t)$ at time $t_{m}$ to the left $x\left(t_{m}-0\right)$. The limit $x\left(t_{m}+0\right)$ of the function $x(t)$ to the right at point $t_{m}$ is computed by solving (14) by $i=m$.

Step $(m+1)$. We solve Cauchy problem (13) in interval $\left(t_{m},+\infty\right)$ under initial condition $x\left(t_{m}\right)=x\left(t_{m}+0\right)$ in order to find the function $x(t)$ in this interval.

It should be noted that the function $M(t)$ due to equality (14) is continuous if we define it as bounds to the left or to the right at points $t_{i}$.

The proposed algorithm is general, but it is extremely hard from the computational point of view. Therefore, for reliability analysis of systems, it is necessary to consider some probability distributions which could simplify the algorithm. One of such probability distributions is the Weibull distribution which is very important and popular in reliability analysis. It has the power function of the cumulative failure rate function. The use of the Weibull probability distribution allows us to get rather simple expressions for computing the reliability measures in the explicit form. 


\section{The Weibull Probability Distribution of Time to Failure}

Let us adapt the proposed algorithm of solving (13) for the case of the Weibull probability distribution and the piecewise smooth function $k(t)$. We assume that the time moments of the load changes are $t_{1}, t_{2}, \ldots, t_{m}, t_{1}<t_{2}<\ldots<t_{m}$.

The reliability function $P(t)$ can be written as $P(t)=$ $e^{-\Lambda(t)}$, where $\Lambda(t)=(t / \beta)^{\alpha}$. Then the reliability function $P_{c}(t)$ can be computed under condition that the following equality holds:

$$
P_{k}(t)=P^{k}(t)
$$

The differential equation (13) for the analyzed case is of the form

$$
x^{\prime}(t)=\frac{k^{\prime}(t)}{k(t)} \frac{t-x(t)}{\alpha} .
$$

It is simple to show that its solution in interval $\left(t_{i}, t_{i+1}\right)$ under initial condition $x\left(t_{i}\right)=x_{i}$ is the following function:

$$
\begin{aligned}
x(t)= & t-k^{-(1 / \alpha)}(t) k^{1 / \alpha}\left(t_{i}+0\right)\left(t_{i}-x_{i}\right) \\
& -k^{-(1 / \alpha)}(t) \int_{t_{i}}^{t} k^{1 / \alpha}(\tau) d \tau .
\end{aligned}
$$

Hence, we can write

$$
\begin{array}{r}
k^{1 / \alpha}(t)(t-x(t))=k^{1 / \alpha}\left(t_{i}+0\right)\left(t_{i}-x_{i}\right)+\int_{t_{i}}^{t} k^{1 / \alpha}(\tau) d \tau, \\
i=1,2, \ldots, m .
\end{array}
$$

In particular, if $t_{i}=0$, then $x_{i}=0$ and the expression (18) is of the form

$$
k^{1 / \alpha}(t)(t-x(t))=\int_{0}^{t} k^{1 / \alpha}(\tau) d \tau
$$
[20].

This is equivalent to the similar expression provided in

By using (18)-(19), we get the function $x(t)$ in the explicit form in intervals of its continuity. The limit values of the function $x(t)$ at the discontinuity points are determined from equalities (14) which can be rewritten now as follows:

$$
k\left(t_{i}+0\right)\left(\frac{t_{i}-x\left(t_{i}+0\right)}{\beta}\right)^{\alpha}=k\left(t_{i}-0\right)\left(\frac{t_{i}-x\left(t_{i}-0\right)}{\beta}\right)^{\alpha} .
$$

At every discontinuity point $t_{i}$ of the load function, we have a linear dependence of the bound to the right and the bound to the left of $x(t)$; that is, there holds

$$
\begin{array}{r}
k^{1 / \alpha}\left(t_{i}+0\right)\left(t_{i}-x\left(t_{i}+0\right)\right)=k^{1 / \alpha}\left(t_{i}-0\right)\left(t_{i}-x\left(t_{i}-0\right)\right), \\
i=1,2, \ldots, m .
\end{array}
$$

The load rate $k(t)$ in interval $\left(0, t_{1}\right)$ is a continuously differentiable function. According to (19) the following expression is valid in this interval:

$$
x(t)=t-k^{-(1 / \alpha)}(t) \int_{0}^{t} k^{1 / \alpha}(\tau) d \tau
$$

Moreover, we can simply find the left-hand limit of the function $x(t)$ at point $t_{1}$ as

$$
k^{1 / \alpha}\left(t_{1}-0\right)\left(t_{1}-x\left(t_{1}-0\right)\right)=\int_{0}^{t_{1}} k^{1 / \alpha}(\tau) d \tau .
$$

It follows from (21) by $i=1$ that the right-hand limit of the function $x(t)$ at point $t_{1}$ can be found as

$$
k^{1 / \alpha}\left(t_{1}+0\right)\left(t_{1}-x\left(t_{1}+0\right)\right)=\int_{0}^{t_{1}} k^{1 / \alpha}(\tau) d \tau .
$$

According to (18), a solution of (13) in interval $\left(t_{1}, t_{2}\right)$ under initial conditions $x\left(t_{1}\right)=x\left(t_{1}+0\right)$ is the function $x(t)$ satisfying the following equality:

$$
\begin{aligned}
k^{1 / \alpha}(t)(t-x(t))= & k^{1 / \alpha}\left(t_{1}+0\right)\left(t_{1}-x\left(t_{1}+0\right)\right) \\
& +\int_{t_{1}}^{t} k^{1 / \alpha}(\tau) d \tau .
\end{aligned}
$$

By using (24), we obtain

$$
\begin{aligned}
k^{1 / \alpha}(t)(t-x(t)) & =\int_{0}^{t_{1}} k^{1 / \alpha}(\tau) d \tau+\int_{t_{1}}^{t} k^{1 / \alpha}(\tau) d \tau \\
& =\int_{0}^{t} k^{1 / \alpha}(\tau) d \tau .
\end{aligned}
$$

It follows from above that the function $x(t)$ has the form of $(22)$ in interval $\left(0, t_{2}\right)$. This implies that the left-hand limit of the function $x(t)$ at point $t_{2}$ satisfies the equality

$$
k^{1 / \alpha}\left(t_{2}-0\right)\left(t_{2}-x\left(t_{2}-0\right)\right)=\int_{0}^{t_{2}} k^{1 / \alpha}(\tau) d \tau \text {. }
$$

It follows from (21) by $i=2$ that the right-hand limit of the function $x(t)$ at point $t_{2}$ can be found as

$$
k^{1 / \alpha}\left(t_{2}+0\right)\left(t_{2}-x\left(t_{2}+0\right)\right)=\int_{0}^{t_{2}} k^{1 / \alpha}(\tau) d \tau .
$$

Let us consider the next interval $\left(t_{2}, t_{3}\right)$. According to (18), the differential equation under the initial condition $x\left(t_{2}\right)=$ $x\left(t_{2}+0\right)$ has the solution $x(t)$, satisfying the equality

$$
\begin{aligned}
k^{1 / \alpha}(t)(t-x(t))= & k^{1 / \alpha}\left(t_{2}+0\right)\left(t_{2}-x\left(t_{2}+0\right)\right) \\
& +\int_{t_{2}}^{t} k^{1 / \alpha}(\tau) d \tau .
\end{aligned}
$$

Then we get due to (28)

$$
\begin{aligned}
k^{1 / \alpha}(t)(t-x(t)) & =\int_{0}^{t_{2}} k^{1 / \alpha}(\tau) d \tau+\int_{t_{2}}^{t} k^{1 / \alpha}(\tau) d \tau \\
& =\int_{0}^{t} k^{1 / \alpha}(\tau) d \tau .
\end{aligned}
$$


Continuing this procedure, we can observe that the function $x(t)$ is defined by (22) for all intervals of time. We can also see that the function $x(t)$ has discontinuities at the same points as the load $k(t)$.

Then the cumulative failure rate for a system with the piecewise smooth load is expressed in the same way as for a system with continuous load in interval $[0,+\infty)$; namely,

$$
M(t)=\Lambda\left(\int_{0}^{t} k^{1 / \alpha}(\tau) d \tau\right)
$$

We have obtained the simple expression for computing the cumulative failure rate and, therefore, the survivor function $P_{c}(t)=e^{-M(t)}$ of the system with an arbitrary piecewise smooth load.

In particular, we can get for the piecewise constant load with constant values $k_{i}$ in intervals $\left(t_{i}, t_{i+1}\right), i=1,2, \ldots, m$, as a special case of the piecewise smooth load,

$$
\begin{array}{r}
\int_{0}^{t} k^{1 / \alpha}(\tau) d \tau=\sum_{j=1}^{i} \int_{t_{j-1}}^{t_{j}} k^{1 / \alpha}(\tau) d \tau+\int_{t_{i}}^{t} k^{1 / \alpha}(\tau) d \tau, \\
t_{i}<t<t_{i+1} .
\end{array}
$$

Then

$$
\int_{0}^{t} k^{1 / \alpha}(\tau) d \tau=\sum_{j=1}^{i} k_{j-1}^{1 / \alpha}\left(t_{j}-t_{j-1}\right)+k_{i}^{1 / \alpha}\left(t-t_{i}\right)
$$

or

$$
\int_{0}^{t} k^{1 / \alpha}(\tau) d \tau=\sum_{j=1}^{i}\left(k_{j-1}^{1 / \alpha}-k_{j}^{1 / \alpha}\right) t_{j}+k_{i}^{1 / \alpha} t
$$

Hence

$$
\begin{array}{r}
M(t)=\left(\frac{\left(k_{i}^{1 / \alpha} t-\sum_{j=1}^{i}\left(k_{j}^{1 / \alpha}-k_{j-1}^{1 / \alpha}\right) t_{j}\right)}{\beta}\right)^{\alpha} \\
\text { by } t_{i} \leq t<t_{i+1} .
\end{array}
$$

By considering the exponential distribution as a special case of the Weibull probability distribution $(\alpha=1)$, we get in this case $M(t)=\Lambda(K(t))$. Here $K(t)=\int_{0}^{t} k(\tau) d \tau$ is the cumulative load function.

\section{Reliability Analysis of Systems under the Monotone Load}

It has been mentioned that the algorithm given in Section 4 is generally extremely hard from computational point of view. The complexity arises when the discrete and continuous loads are combined. Therefore, special software has been developed for implementing this algorithm. The corresponding software program realizes approximate methods. At the same time, the numerical experiments with some special cases including the use of the Weibull probability distribution of time to failure illustrated the high quality of the developed software.

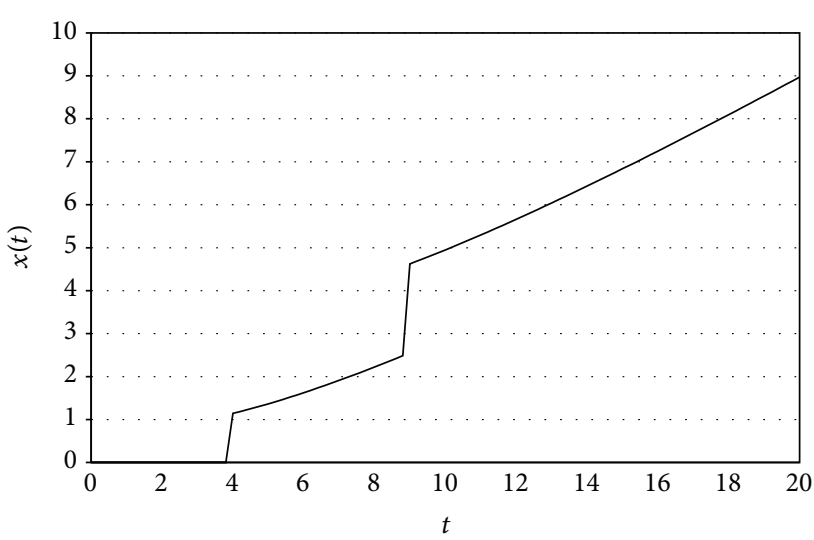

Figure 1: The function $x(t)$.

We will show below by means of three numerical examples how the reliability measures of a system without load and with the monotone load depend on the type of the probability distribution of system time to failure. The parameters of probability distributions in all examples are identical, in particular, and the mean value is $m=10 \mathrm{~h}$ and the standard deviation is $\sigma=2 \mathrm{~h}$. The exponential distribution has one parameter.

Let us suppose that the system is under the nondecreasing load with the load rate

$$
k(t)= \begin{cases}1, & t<4 \\ 1+0.1 t, & 4 \leq t \leq 9 \\ 1+0.2 t, & t>9\end{cases}
$$

One can see that the load function has discontinuities of the first kind at points $4 \mathrm{~h}$ and $9 \mathrm{~h}$. The size of the first jump of the function $k(t)$ at time $t=4$ is equal to 0.4 . The size of the second jump at $t=9$ is 0.9 . We compute the survivor function $P_{c}(t)$ for different probability distributions of time to failure of the system without load.

Example 1. It is assumed that time to failure is governed by the exponential probability distribution with the failure rate $\lambda=0.1 \mathrm{~h}^{-1}$. We compute $P_{c}(t)$ by using the exact expression and by using the software developed for comparison of results. First, we compute the cumulative load function defined as $K(t)=\int_{0}^{t} k(\tau) d \tau$. It is obvious that there holds

$$
K(t)= \begin{cases}t, & t<4 \\ 0.05 t^{2}+t-0.8, & 4 \leq t \leq 9 \\ 0.1 t^{2}+t-4.85, & t>9\end{cases}
$$

The function $x(t)$ for the exponential probability distribution can be written as $x(t)=t-K(t) / k(t)$. Hence, there holds $P_{c}(t)=e^{-0,1 K(t)}$. The values of $P_{c}(t)$ computed in two ways and values of other auxiliary reliability measures are given in Table 1.

The function $x(t)$ is shown in Figure 1 .

One can see from Table 1 that the values of $P_{c}(t)$ computed by means of the exact expression and by the software coincide 
TABLE 1: The values of reliability measures as functions of time.

\begin{tabular}{|c|c|c|c|c|c|c|}
\hline \multirow{2}{*}{$t$} & \multirow{2}{*}{$P(t)$} & \multirow{2}{*}{$k(t)$} & \multirow{2}{*}{$K(t)$} & \multirow{2}{*}{$x(t)$} & \multicolumn{2}{|c|}{$P_{c}(t)$} \\
\hline & & & & & Exact & Software \\
\hline 0 & 1 & 1 & 0 & 0 & 1.0000 & 1.0000 \\
\hline 1 & 0.9048 & 1 & 1.00 & 0 & 0.9048 & 0.9048 \\
\hline 2 & 0.8187 & 1 & 2.00 & 0 & 0.8187 & 0.8187 \\
\hline 3 & 0.7408 & 1 & 3.00 & 0 & 0.7408 & 0.7408 \\
\hline 4 & 0.6703 & 1.4 & 4.00 & 1.1429 & 0.6703 & 0.6703 \\
\hline 5 & 0.6065 & 1.5 & 5.45 & 1.3667 & 0.5798 & 0.5798 \\
\hline 6 & 0.5488 & 1.6 & 7.00 & 1.6250 & 0.4966 & 0.4966 \\
\hline 7 & 0.4966 & 1.7 & 8.65 & 1.9118 & 0.4211 & 0.4211 \\
\hline 8 & 0.4493 & 1.8 & 10.4 & 2.2223 & 0.3535 & 0.3535 \\
\hline 9 & 0.4066 & 2.8 & 12.25 & 4.6251 & 0.2938 & 0.2938 \\
\hline 10 & 0.3679 & 3.0 & 15.15 & 4.9500 & 0.2198 & 0.2198 \\
\hline 11 & 0.3329 & 3.2 & 18.25 & 5.2969 & 0.1612 & 0.1612 \\
\hline 12 & 0.3012 & 3.4 & 21.55 & 5.6618 & 0.1159 & 0.1159 \\
\hline 13 & 0.2725 & 3.6 & 25.05 & 6.0417 & 0.0817 & 0.0817 \\
\hline 14 & 0.2466 & 3.8 & 28.75 & 6.4342 & 0.0564 & 0.0564 \\
\hline 15 & 0.2231 & 4.0 & 32.65 & 6.8375 & 0.0382 & 0.0382 \\
\hline 16 & 0.2019 & 4.2 & 36.75 & 7.2500 & 0.0253 & 0.0253 \\
\hline 17 & 0.1827 & 4.4 & 41.05 & 7.6705 & 0.0165 & 0.0165 \\
\hline 18 & 0.1653 & 4.6 & 45.55 & 8.0979 & 0.0105 & 0.0105 \\
\hline 19 & 0.1496 & 4.8 & 50.25 & 8.5313 & 0.0066 & 0.0066 \\
\hline 20 & 0.1353 & 5.0 & 55.15 & 8.9700 & 0.0040 & 0.0040 \\
\hline
\end{tabular}

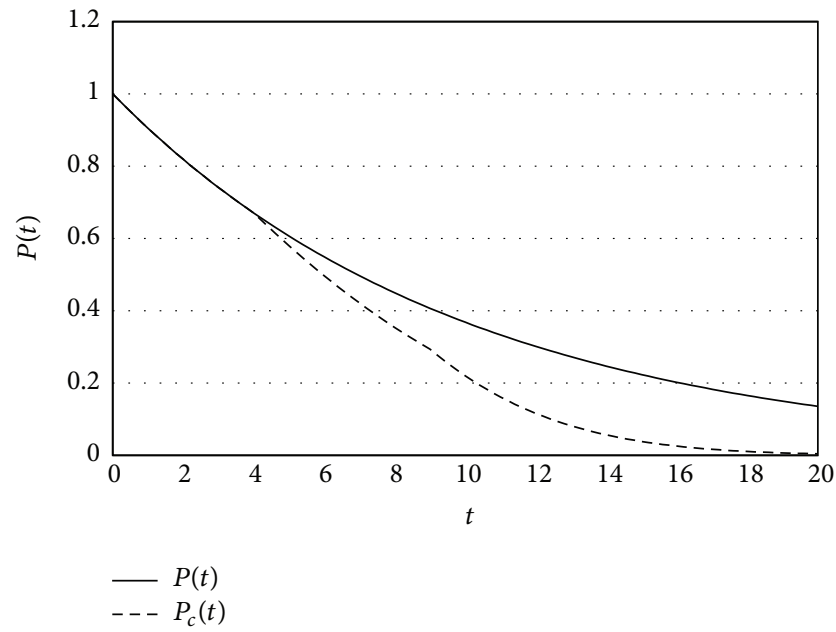

Figure 2: Survivor functions $P(t)$ and $P_{c}(t)$.

up to 4 digits after comma. Survivor functions of the system without load $P(t)$ and under the load $P_{c}(t)$ are depicted in Figure 2.

The mean time to failure of the system without load is $T_{1}=10 \mathrm{~h}$ and that of the same system under the load is $T_{1 c}=6.50 \mathrm{~h}$.

One can see that the change of the system load leads to decreasing the system reliability. Moreover, it is clear to observe from Figure 2 that the survivor function quickly

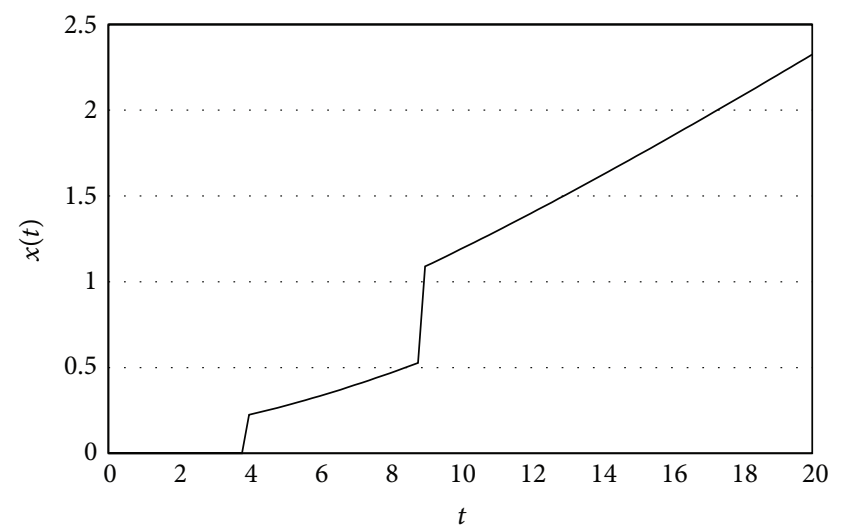

Figure 3: The function $x(t)$.

decreases after time $t=4$, that is, after the first change of the load.

Example 2. It is assumed that time to failure is governed by the Weibull distribution with $m=10 \mathrm{~h}$ and $\sigma=2 \mathrm{~h}$. Values of the function $x(t)$ can be simply computed by using the expression (22). The survivor functions can be again obtained my means of the explicit expression (31) and the software. The function $x(t)$ is depicted in Figure 3.

Survivor functions of the system without load $P(t)$ and under the load $P_{c}(t)$ are depicted in Figure 4. 


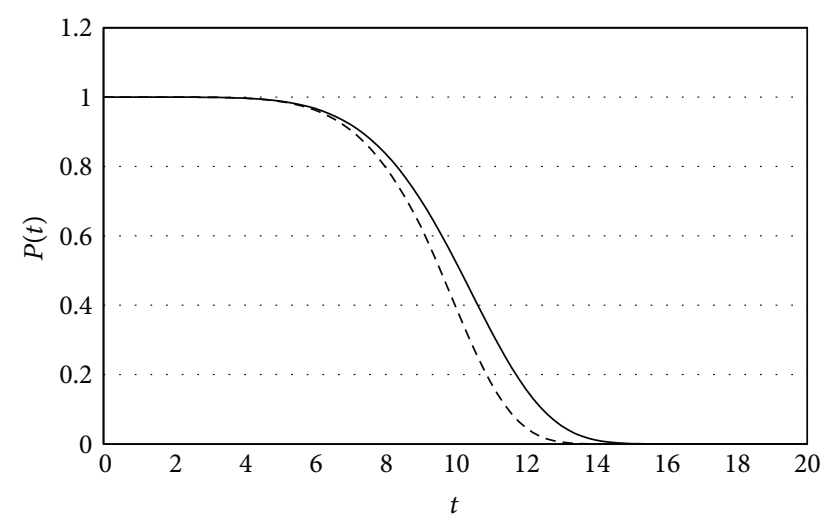

$-P(t)$

$--P_{c}(t)$

FIgure 4: Survivor functions $P(t)$ and $P_{c}(t)$.

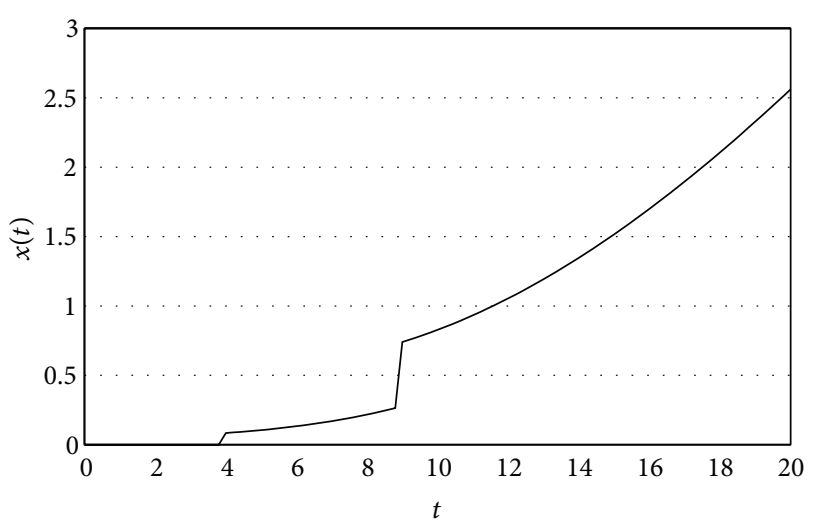

FIGURE 5: The function $x(t)$.

The mean time to failure of the system without load is $T_{1}=10 \mathrm{~h}$ and that of the same system under the load is $T_{1 c}=9.41 \mathrm{~h}$.

Example 3. It is assumed that time to failure is governed by the Gamma probability distribution with $m=10 \mathrm{~h}$ and $\sigma=$ $2 \mathrm{~h}$. The function $x(t)$ is depicted in Figure 5. We see stepwise changes of the function $x(t)$. Survivor functions $P(t)$ and $P_{c}(t)$ are depicted in Figure 6 . The mean time to failure of the system without load is $T_{1}=10 \mathrm{~h}$ and that of the same system under the load is $T_{1 c}=9.1 \mathrm{~h}$.

\section{Reliability Analysis of Systems under the Nonmonotone Load}

We will show below by means of two numerical examples how the reliability measures of a system without load and with the nonmonotone load depend on the type of the probability distribution of system time to failure.

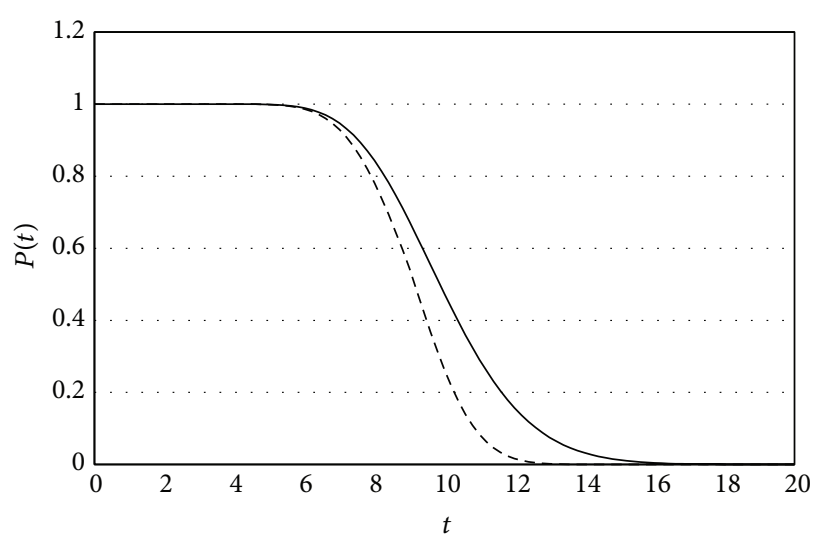

$-P(t)$
$---P_{c}(t)$

Figure 6: Survivor functions $P(t)$ and $P_{c}(t)$.

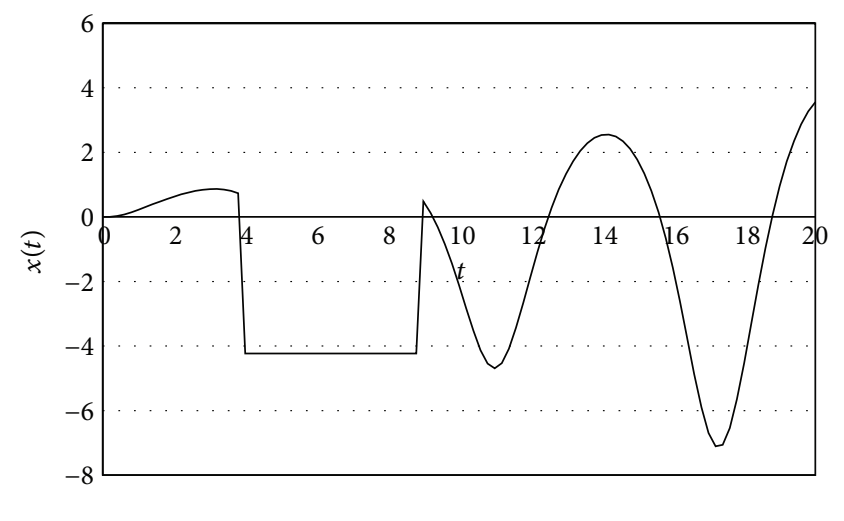

Figure 7: The function $x(t)$.

Let us suppose that the system is under the nonmonotone load with the load rate

$$
k(t)= \begin{cases}4+3 \sin \left(t-\frac{\pi}{2}\right), & t<4 \\ 1, & 4 \leq t \leq 9 \\ 2+\sin t, & t>9 .\end{cases}
$$

One can see that the load function has discontinuities of the first kind at points $4 \mathrm{~h}$ and $9 \mathrm{~h}$. We again compute the survivor function $P_{c}(t)$ for different probability distributions of time to failure of the system without load.

Example 4. It is assumed that time to failure is governed by the Rayleigh distribution with the mean value $m=10 \mathrm{~h}$. The function $x(t)$ is depicted in Figure 7. Survivor functions $P(t)$ and $P_{c}(t)$ are shown in Figure 8.

The mean time to failure of the system without load is $T_{1}=10 \mathrm{~h}$ and that of the same system under the load is $T_{1 c}=6.3 \mathrm{~h}$.

Example 5. It is assumed that time to failure is governed by the normal distribution with the mean value $m=10 \mathrm{~h}$ and the standard deviation $\sigma=2 \mathrm{~h}$. 


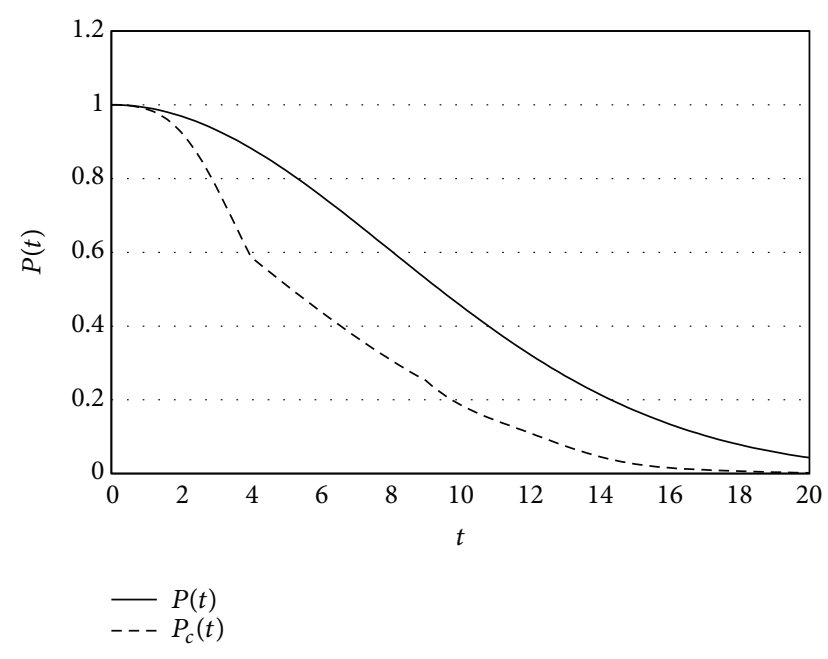

Figure 8: Survivor functions $P(t)$ and $P_{c}(t)$.

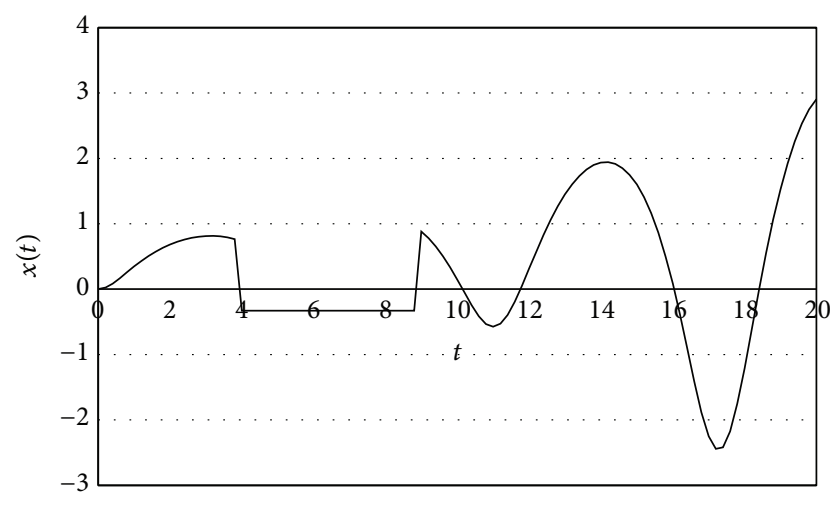

Figure 9: The function $x(t)$.

The function $x(t)$ is depicted in Figure 9. Survivor functions $P(t)$ and $P_{c}(t)$ are shown in Figure 10. The mean time to failure of the system without load is $T_{1}=10 \mathrm{~h}$ and that of the same system under the load is $T_{1 c}=9.52 \mathrm{~h}$.

It follows from the Figures 7 to 10 that there is a fundamental difference between the reliability measures of system under the load for the Rayleigh and normal distributions. The functions $P(t)$ and $P_{c}(t)$ are rather close to each other for the normal distribution, but they differ significantly from each other for the Rayleigh distribution. In spite of the difference between survivor functions, the functions $k(t)$ are very similar. They have the same intervals of constancy and fluctuations. One can see also the significant difference between mean times to failure.

It is interesting to compare the obtained results for the nonmonotone load with the similar results in the paper [20, Example 6.10] where the normal distribution with the same parameters was used. The load rate in this example had the oscillatory behavior $k(t)=7+6 \sin (t-\pi / 2)$. It can be seen from the example that the survivor functions are very similar. Moreover, the mean time to failure of the system under the load is also close and $T_{1 c}=9.66 \mathrm{~h}$. This implies that the probability distribution of time to failure strongly impacts

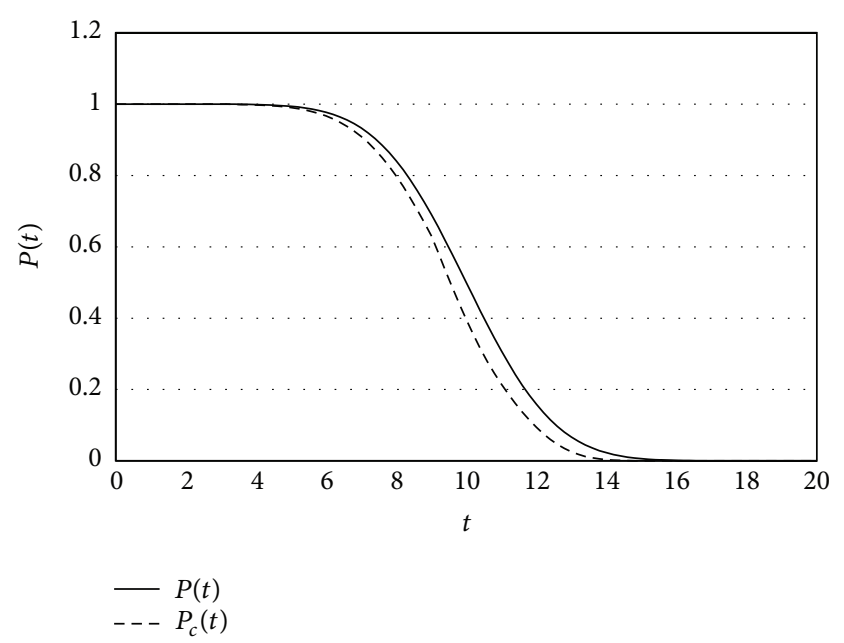

FIgURE 10: Survivor functions $P(t)$ and $P_{c}(t)$.

on the reliability behavior of a system, but the piecewise smooth load function can be replaced by its continuous approximation in some applications.

\section{Comparison of Systems under Piecewise Constant and Smooth Loads}

Let us compare the obtained results with the reliability measures of systems by discrete load when changes occur step-wise at discrete time instances and the loads between these instances are constant. At that, we take examples from the previous sections with the same parameters, but the load rate is step-wise at instances coinciding with discontinuity points $t_{i}$.

Example 6. Let us suppose that the system is under the nondecreasing step-wise load with the load rate

$$
k(t)= \begin{cases}1, & t<4 \\ 1.4, & 4 \leq t \leq 9 \\ 2.8, & t>9\end{cases}
$$

It is assumed that time to failure is governed by the Gamma probability distribution with $m=10 \mathrm{~h}$ and $\sigma=2 \mathrm{~h}$ (see Example 3 for comparison). The function $x(t)$ is depicted in Figure 11. We see step-wise changes of the function $x(t)$. It can be seen from the figure that the function $x(t)$ is also constant between discontinuity time points. Survivor functions $P(t)$ and $P_{c}(t)$ are depicted in Figure 12. The mean time to failure of the system under the load is $T_{1 c}=9.26 \mathrm{~h}$. It is larger than the same reliability measure obtained in Example $3\left(T_{1 c}=9.1 \mathrm{~h}\right)$.

Example 7. Let us suppose that the system is under the nonmonotone step-wise load with the load rate

$$
k(t)= \begin{cases}1, & t<4 \\ 0.5, & 4 \leq t \leq 9 \\ 2, & t>9 .\end{cases}
$$




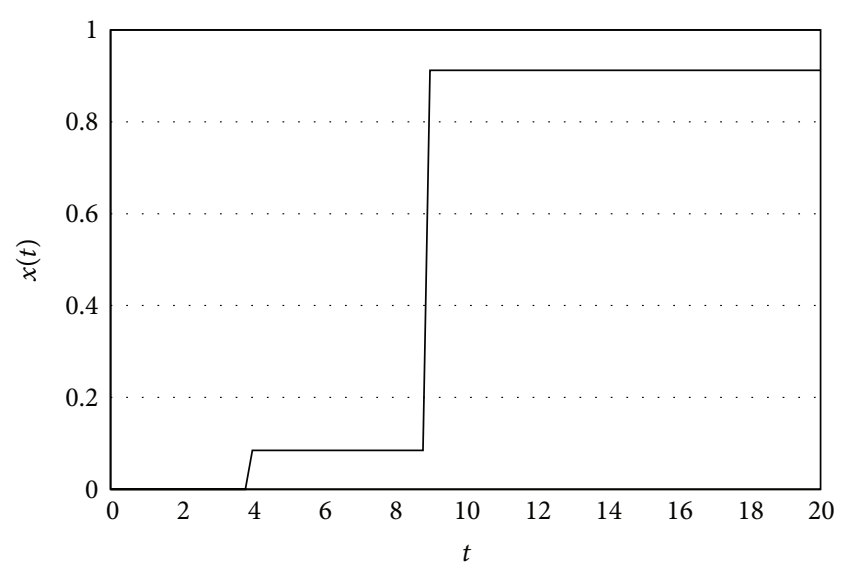

FIGURE 11: The function $x(t)$.

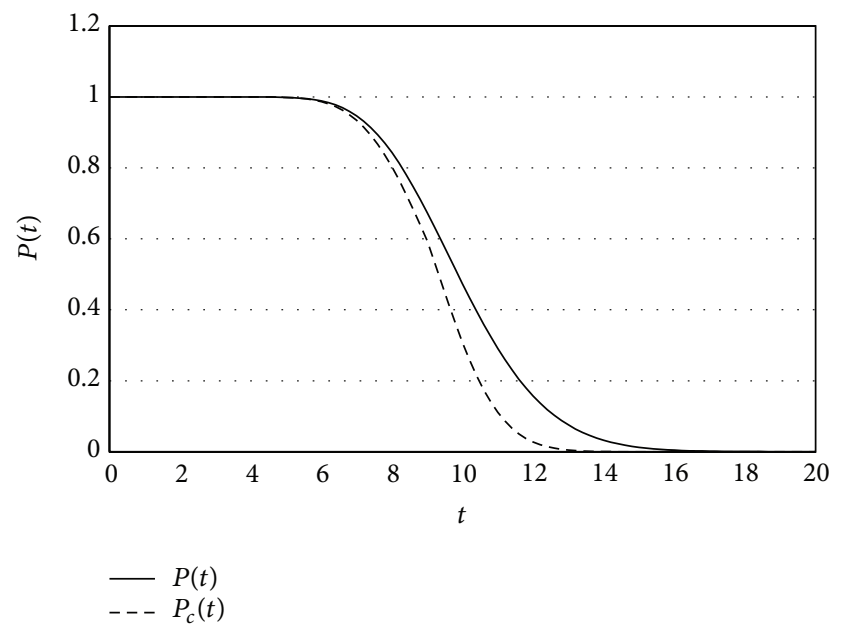

FIGURE 12: Survivor functions $P(t)$ and $P_{c}(t)$.

It is assumed that time to failure is governed by the normal distribution with the mean value $m=10 \mathrm{~h}$ and the standard deviation $\sigma=2 \mathrm{~h}$ (see Example 5 for comparison).

The function $x(t)$ is depicted in Figure 13. It should be noted that this function is negative in interval between $4 \mathrm{~h}$ and $9 \mathrm{~h}$ and it is positive after $9 \mathrm{~h}$. This means that the system reliability between $4 \mathrm{~h}$ and $9 \mathrm{~h}$ is smaller than the reliability of the same system without load. This is also seen from Figure 14, where survivor functions $P(t)$ and $P_{c}(t)$ are depicted.

The mean time to failure of the system under the load is $T_{1 c}=10.19 \mathrm{~h}$. This reliability measure is larger than the same measure obtained in Example $5\left(T_{1 c}=9.52 \mathrm{~h}\right)$. Moreover, it is interesting to note that the obtained mean time to failure is also larger than the same measure of the system without load which is $T_{1}=10 \mathrm{~h}$.

\section{Conclusion}

The reliability analysis of systems under the changeable piecewise smooth load as a special case of the piecewise constant load and continuous load has been provided in the

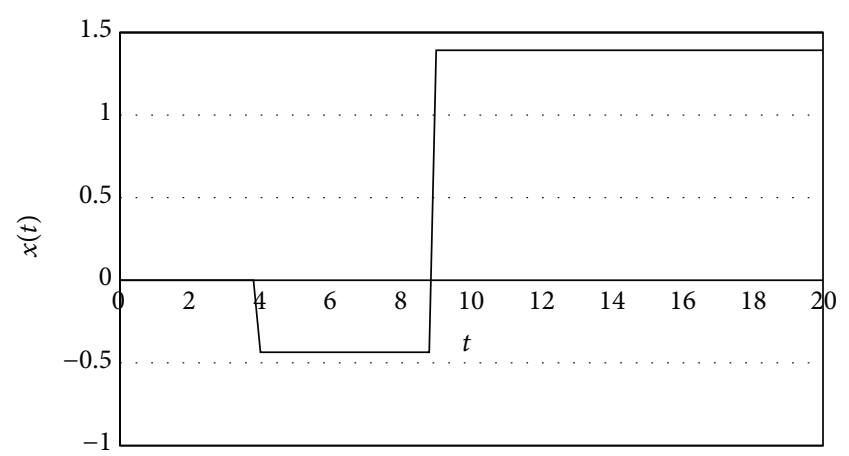

Figure 13: The function $x(t)$.

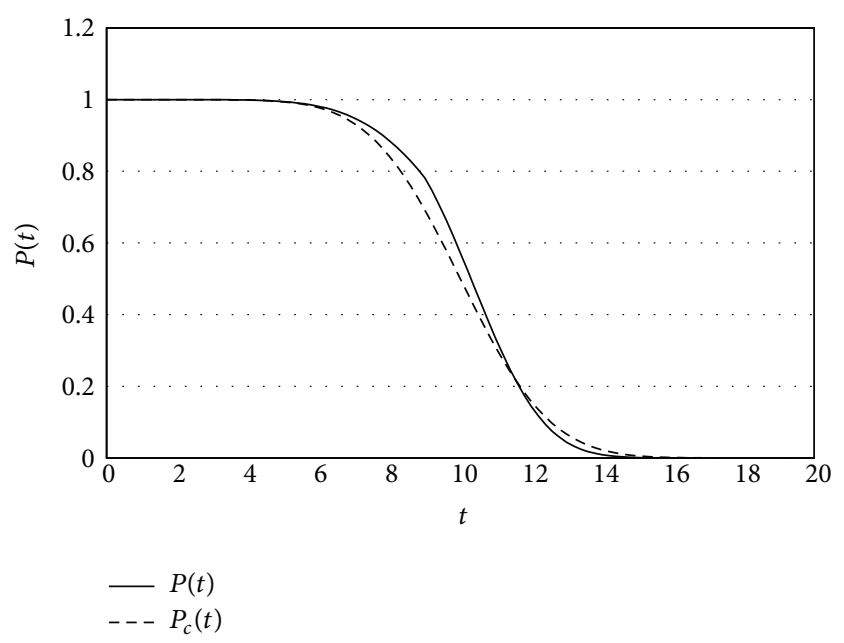

FIgURE 14: Survivor functions $P(t)$ and $P_{c}(t)$.

paper. The main feature of the considered models is applying the condition of the residual lifetime conservation to reliability analysis which is crucial in the reliability models. The condition means that the cumulative distribution function of time to failure is continuous; that is, it cannot have jumps. Another important condition is that the change of the load in $k$ times leads to change of the system failure rate in $k$ times. By accepting these conditions, the reliability analysis is reduced to solving the differential equations of a special form for computing the reliability measure such that the survivor function, the mean time to failure, and so forth.

A special case of the Weibull probability distribution of time to failure has been considered in detail. In this case, the solution to the complex differential equations in the explicit form can be found. This fact significantly simplifies the reliability analysis.

Various numerical examples have illustrated how the different forms of the system load and different probability distributions of time to failure can impact on the system reliability. Moreover, the examples have shown that the reliability analysis can be carried out even by very complex function of the load. 


\section{Conflict of Interests}

The authors declare that there is no conflict of interests regarding the publication of this paper.

\section{References}

[1] P. H. Kvam and E. A. Peña, "Estimating load-sharing properties in a dynamic reliability system," Journal of the American Statistical Association, vol. 100, no. 469, pp. 262-272, 2005.

[2] M. Meldorf, Ü. Treufeldt, and J. Kilter, "Temperature dependency of electrical network load," Oil Shale, vol. 24, no. 2, pp. 237-247, 2007.

[3] E. Valor, V. Meneu, and V. Caselles, "Daily air temperature and electricity load in Spain," Journal of Applied Meteorology, vol. 40, no. 8, pp. 1413-1421, 2001.

[4] M. Tanrioven and M. S. Alam, "Reliability modeling and assessment of grid-connected PEM fuel cell power plants," Journal of Power Sources, vol. 142, no. 1-2, pp. 264-278, 2005.

[5] H. E. Daniels, "The statistical theory of the strength of bundles of threads," Proceedings of the Royal Society A: Mathematical, Physical and Engineering Sciences, vol. 183, pp. 405-435, 1945.

[6] M. Bebbington, C. Lai, and R. Zitikis, "Reliability of modules with load-sharing components," Journal of Applied Mathematics and Decision Sciences, vol. 2007, Article ID 43565, 18 pages, 2007.

[7] J. V. Deshpande, I. Dewan, and U. V. Naik-Nimbalkar, "A family of distributions to model load sharing systems," Journal of Statistical Planning and Inference, vol. 140, no. 6, pp. 1441-1451, 2010.

[8] S. Lee, S. Durham, and J. Lynch, "On the calculation of the reliability of general load sharing systems," Journal of Applied Probability, vol. 32, no. 3, pp. 777-792, 1995.

[9] H. Kim and P. H. Kvam, "Reliability estimation based on system data with an unknown load share rule," Lifetime Data Analysis, vol. 10, no. 1, pp. 83-94, 2004.

[10] J. D. Lynch, "On the joint distribution of component failures for monotone load-sharing systems," Journal of Statistical Planning and Inference, vol. 78, no. 1-2, pp. 13-21, 1999.

[11] S. M. Ross, "A model in which component failure rates depend on the working set," Naval research logistics quarterly, vol. 31, no. 2, pp. 297-300, 1984.

[12] J. Shao and L. R. Lamberson, "Modeling a shared-load k-out-ofn:g system," IEEE Transactions on Reliability, vol. 40, no. 2, pp. 205-209, 1991.

[13] C. Stefanescu and B. W. Turnbull, "Multivariate frailty models for exchangeable survival data with covariates," Technometrics, vol. 48, no. 3, pp. 411-417, 2006.

[14] K. Yang and H. Younis, "A semi-analytical Monte Carlo simulation method for system's reliability with load sharing and damage accumulation," Reliability Engineering and System Safety, vol. 87, no. 2, pp. 191-200, 2005.

[15] C. Park, "Parameter estimation for the reliability of load-sharing systems," IIE Transactions (Institute of Industrial Engineers), vol. 42, no. 10, pp. 753-765, 2010.

[16] C. Park, "Parameter estimation from load-sharing system data using the expectation-maximization algorithm," IIE Transactions, vol. 45, no. 2, pp. 147-163, 2013.

[17] B. Singh and P. K. Gupta, "Load-sharing system model and its application to the real data set," Mathematics and Computers in Simulation, vol. 82, no. 9, pp. 1615-1629, 2012.
[18] V. Volovoi, "Universal failure model for multi-unit systems with shared functionality," Reliability Engineering and System Safety, vol. 119, pp. 141-149, 2013.

[19] S. V. Gurov and L. V. Utkin, "Load-share reliability models with the piecewise constant load," International Journal of Reliability and Safety, vol. 6, no. 4, pp. 338-353, 2012.

[20] S. V. Gurov and L. V. Utkin, "A continuous extension of a load-share reliability model based on a condition of the residual lifetime conservation," European Journal of Industrial Engineering, vol. 8, no. 3, pp. 349-365, 2014. 

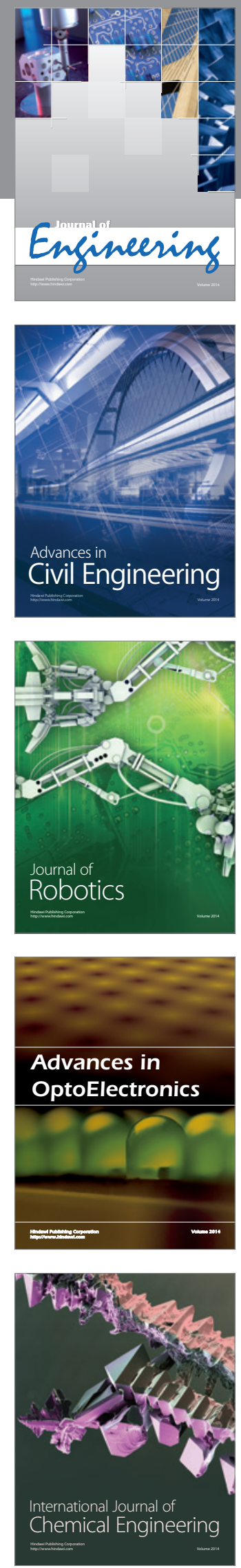

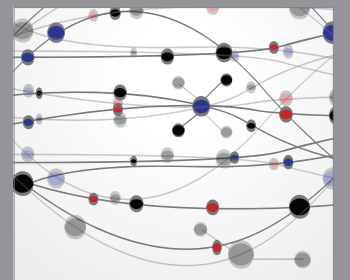

The Scientific World Journal
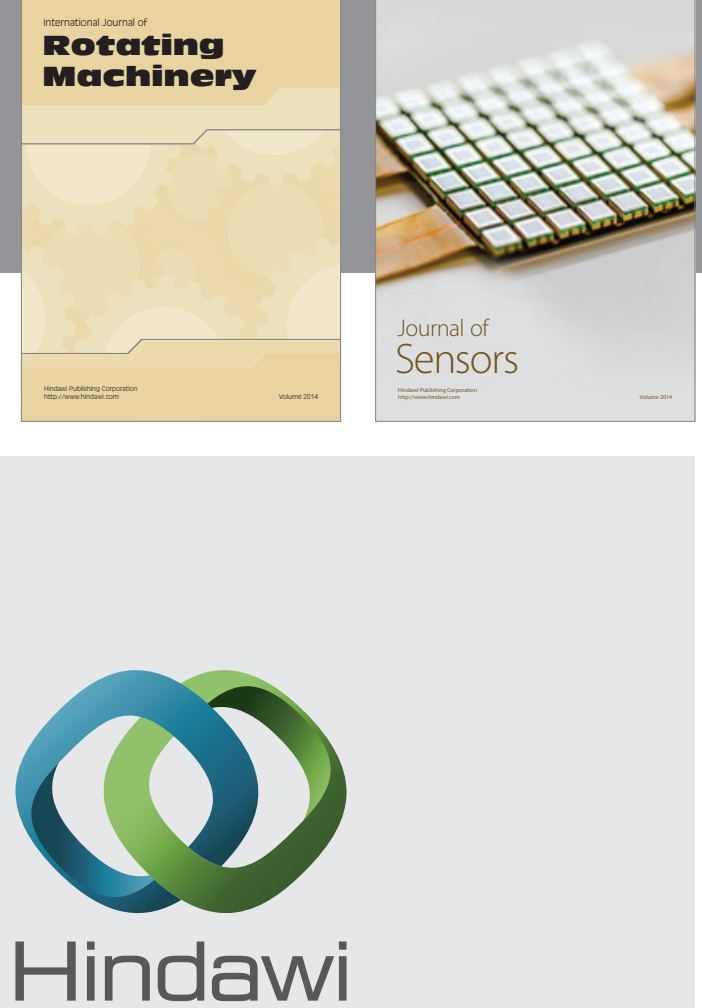

Submit your manuscripts at http://www.hindawi.com
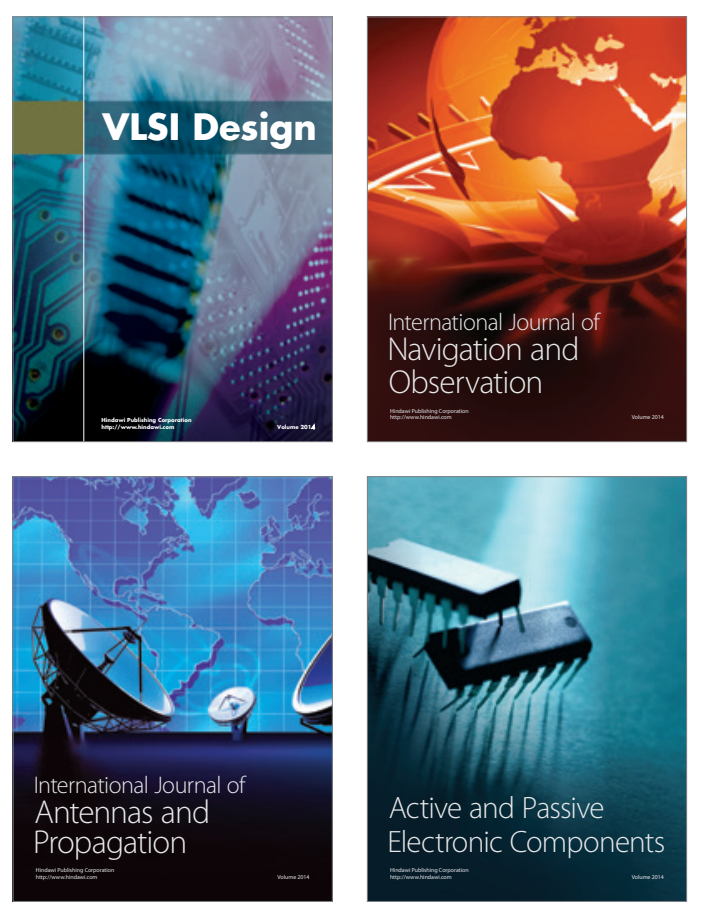
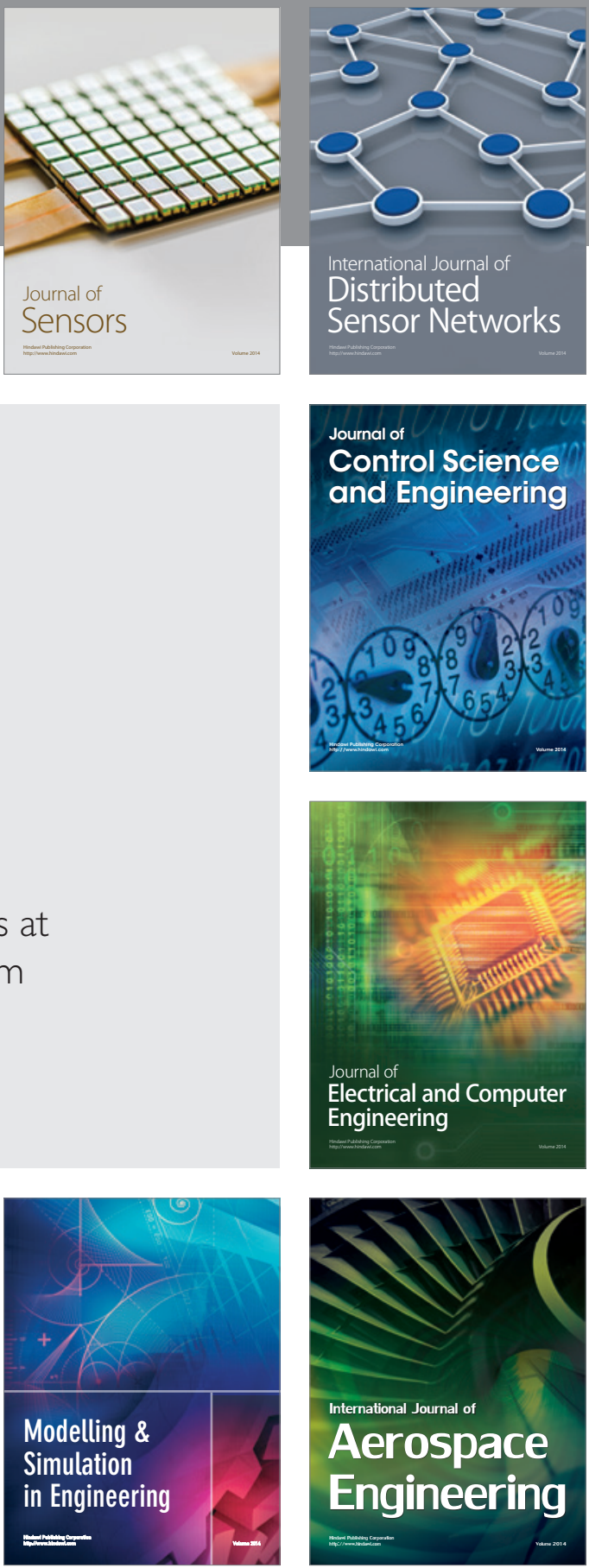

Journal of

Control Science

and Engineering
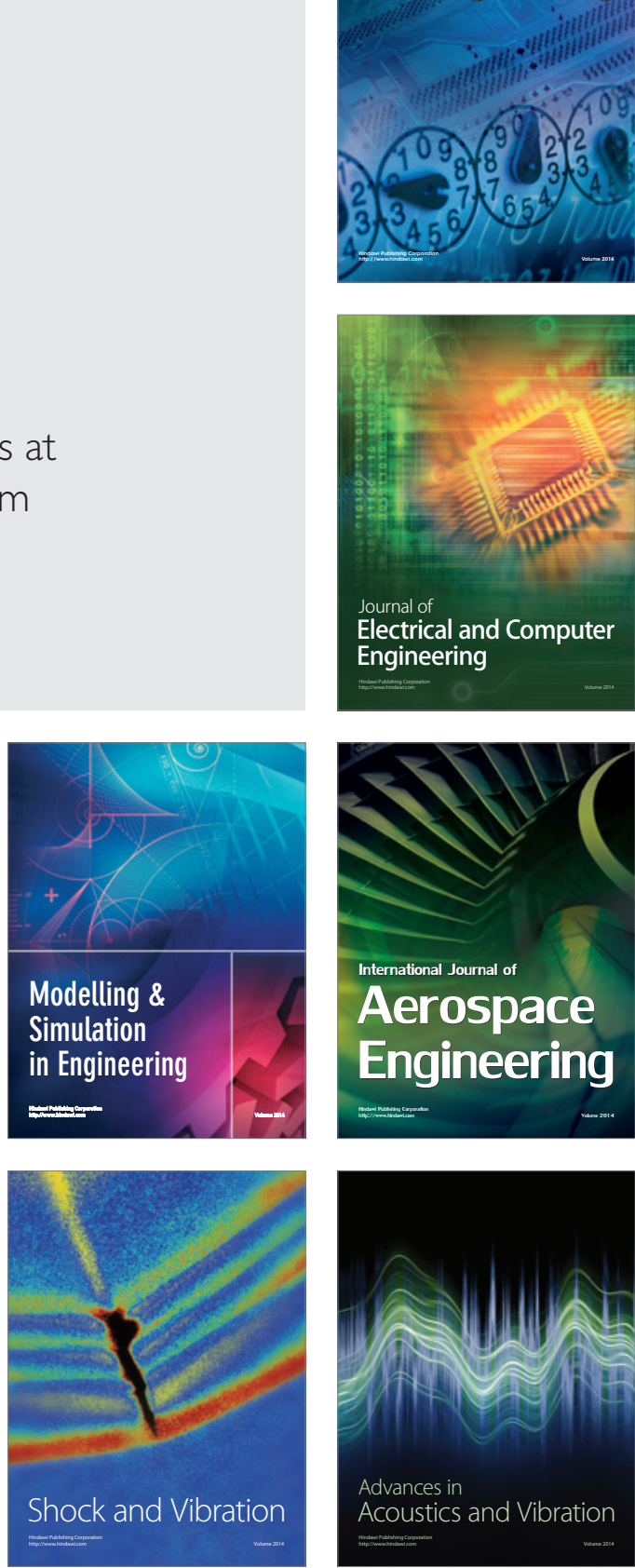